\title{
Blockchain Technology-Based Medical Information System for Personalized Medical Services
}

\author{
Sang Young Lee \\ Department of Health Administration, Namseoul University, South Korea \\ sylee@nsu.ac.kr
}

\begin{abstract}
Blockchain technology can be used throughout health and medical field. In particular, we need to pay attention to the fact, which medical consumers, that is, individuals who were recognized as a victim of information asymmetry due to the medical information monopoly of hospitals can regain their sovereignty through blockchain technology. In this paper, we suggested a framework that can complete Personal Health Record (PHR) system, using blockchain technology. The current medical paradigm is shifting from treatment to management. In the medical management, it is important to know not only the current health condition of the patients but also the existing disease and its treatment process. The storage method of medical information is changing to PHR, an integrated record of EMR information, which are additional medical information of patients beyond the environment where medical information is recorded and stored within the EMR system. The emergence of PHR, according to such change in the medical field, makes it easy to understand the health condition of patients. Therefore, it is suitable for providing personalized medical services to patients.
\end{abstract}

Keywords: Personal Health Record (PHR), Blockchain, medical, modelling. 


\section{Introduction}

With the constant advancement in ICT, there have been various improvement on the healthcare devices and services. A number of companies have provided new healthcare value to doctors and patients using state-of-the-art technology such as sensor, smartphone, wireless communication, etc (Richard et al., 2016 and Steve et al., 2016). There have been growing efforts on the use of smart devices such as smartphones and health devices for patients and healthy individuals to take care of themselves and prevent diseases. In other words, interest in and usage of the Personal Health Record (PHR) system, which presents a new health value to those working in medical sector through innovative companies that develop these technologies, are growing more and more (Marlon et al., 2018 and Tuan et al., 2017). This trend allows not only patients but also healthy people to monitor their health conditions using the said smart IT devices (Zibin et al., 2017; Zimmer, 2017; European Parliament, 2016).

The PHR can be recognized as decisive tool for making partnerships between healthcare providers and consumers. It is also expected to reduce or remove duplicate treatment or medical procedures and save cost and time by using PHR system. Since patients can better understand their health information through this, they can active role in preventive healthcare and disease control, increasing the rights of patients (FCA, 2017 and Yli-Huumo et al., 2016).

Blockchain technology is a possible method for setting security for PHR. It is also a good way to solve problems with a distributed PHR system. Blockchain technology was initially utilized in the financial sphere using virtual currencies. And in recent years, it has been studied and implemented in various fields, such as in the field of health. Various approaches have been proposed for applying blockchain to health data, mainly focusing on constructing decentralized health records and providing useful tools to protect patient privacy. The performance of decentralized PHRs and the integration of health data between medical institutions are an important factor in the adoption of blockchain technology (Hölbl et al., 2016; Park et al., 2019; Roehrs et al., 2016).

In this healthcare paradigm shift, it is important to record ordinary and periodic information on health condition as well as underlying diseases and information on treatment process. The form of medical information is changing from the environment where medical information is recorded and saved by the EMR system to environment where it is saved by the PHR, personal health information of patients (Lee, 2020, and Sekhar and Suneetha, 2018). This PHR is very useful to understand the health condition of patients, making it suitable to provide personalized medical service to patients. However, it is difficult to to complete the information with the PHR because the EMR system is created differently for each medical institution and the medical information is integrated accordingly (Kim et al., 2018 and Naga Mallik Raj and Neeraja). The cloud can be a suitable alternative 
since it is easy to build and process integrated PHR while maintaining the system under various EMR systems. In addition, it can solve security and stability issues in the sensitive part of medical information through blockchain. If PHR is built in the Cloud using blockchain, medical information can be used widely while maintaining the current medical system. In addition, it applies distributed data process algorithm using blockchain on high costs resulted from the management of individual's data under the cloud environment. This method allows minimizing the costs for individual. The medical information system will provide positive impact on the medical community and to provide appropriate medical services to patients. Therefore, this paper presents a framework that can complete the information management within the PHR system using blockchain technology.

\section{Related Works}

The main difference between HER and PHR is that PHR allows patients to access and control their own data. Basically, PHR is a new trend with innovative potential in the field of healthcare. We must focus on improving the management and sharing of health records and take into account environmental factors (Jang et al., 2016 and Fragidis and Chatzoglou, 2017).

Initiatives to adopt PHR have evolved in recent years, but there are challenges in terms of security. Therefore, these applications face many challenges for adoption. And another challenge facing PHR is the distribution and limitation of health record consolidation. Another issue concerns security issues such as the confidentiality and privacy of health records. Patient health data is usually stored in the health care provider's repository (Chiauzzi et al., 2015 and Nakamoto, 2008). However, often these data are not shared with providers or patients. In addition, even where there is an intention to share data, there are problems in achieving the goals, including the following (Zhang et al., 2017 and Randall et al., 2017)

-Interoperability due to lack of common health data standards.

-Difficulty in consolidating large amounts of data contained in medical records.

PHR contains personally managed health information. Often the term patient is used to refer to a stakeholder in PHR. However, PHR is a tool that can help maintain health and well-being. And I prefer to use the term individual to emphasize that these tools are tools that help with disease.

This can be contrasted with the clinician's record of patient encounter-related information (also called a paper chart or EHR, electronic medical record, or computer-based patient record) maintained by a doctor or medical institution. Over time, when a patient approves such sharing, it creates an environment in which health information about an individual can move seamlessly between the systems used by certified healthcare professionals, caregivers, and patients.

Blockchain is composed of block lists connected with chains. Such blockchain is distributed through peer to peer (P2P) networks with the latest version of all nodes. 
The block is a record of transaction data. Blockchain based on Bitcoin is one of distributed ledger technologies, which is a distributed, shared, and encrypteddatabase that serves as an "irreversible and incorruptible repository of information". The block includes header and body.

- Version of Block: It represents validation rules that are based on a series of blocks.

- Parent Block Hash: It is 256-bit hash speed.

- Merkle Tree Root Hash: Hash of all the hashed of all the transactions.

- Timestamp: Currently, it represents the value for every second.

- nBits: it consists of simple target hash.

This field is divided into electronic records-based EMR, interchange-based HER and personal health record-based PHR[2, 3]. The main research in related fields so far were about the management of personal medical or health related data for generating and storing electronic documents. In fact, the research on cases of blockchain EMR use were focused on decentralization, immutability, data sources, reliability, rigidity, contract, security and privacy of blockchain. The focus here is on research on the use of blockchain for storing and managing electronic medical records (EMR) of patients and methods to facilitate data sharing centered on patients in various medical services. In particular, researchers on sharing, processing, and using of technology and data that build medical platform, allowing patients to control their own methods[6, 7, 8]. Applications using blockchain technology for managing healthcare data are introducing blockchain to apply perfect security technology in designing medical systems[9, 12].

In this paper, we present architecture for gateway application of healthcare data for easy and safe control and sharing. In other words, we present to use blockchain technology using multi-step authentication to safely protect and share the medical data between different objects that can use the data of patients. In addition, we present research scenarios related to biometrics and biomedicine for the security of medical data.

\section{PHR and standards}

EMR and EHR are the latest buzzwords in the health industry. A recent medical survey found that only $7 \%$ of doctors knew the real difference between these two terms. EMR, or electronic medical record, refers to a digital version of a patient chart with information stored on a computer system. Thus, all the patient's papers, such as his medical history, laboratory tests, diagnosis, etc., are stored in the system rather than in bulky paper file format. This actually works fine, but is limited as you can't get out of the premises of the organization.

This is where the real difference between EHR and EMR comes into play. An EHR or electronic health record is a person's digital health information. It contains a lot more than what is already included in the EMR. EHR includes vital signs, past 
medical history, diagnosis, progress, medications, allergies, laboratory data, vaccination dates and imaging reports. This information can also be moved outside the organization's premises.

As self-monitoring technologies become more feasible and cheaper, medical technologists are using tools such as personal health records (PHR) to help patients better connect with their health data. PHR is a technology application that creates a private and secure environment where users can access, manage and share medical data. PHR is essential for patients to manage their medical information and to serve as an engaging tool for patients to interact with their healthcare professionals. The main consumers of PHR are patients. The goal of PHR is to process patient health information so that patients can participate in their treatment and promote faster and better communication between patients and providers.

PHR falls into two categories. Tethering and untethering. The ability to exchange information with other health systems, such as electronic health records (EHR), is called tethered PHR. While data entry for tethered PHRs is automated, non-tethered PHRs cannot be integrated, so data must be entered manually. PHR plays an important role in transforming healthcare services using health information technology. Easily access to information via the Internet has increased patient awareness of the treatment process.

In the United States, $42 \%$ of the population maintain personal records in paper format, but only $7 \%$ of American adults use PHR. Although the PHR system is an integral part of the current health care system, barriers to adoption must still be overcome. Reasons for lack of adoption include lack of interest, functional interpretation, issues of education, usability, and lack of knowledge in the consumer and health sectors. Overall, health care has evolved into a more personalized management approach as the way health data is accessed improves and users engage in decision making.

The development of hospital information system, the spread of computerized medical records, and simplification of treatment and its support process increase the efficiency in terms of economy and time. Convergence with information and communication technology for outpatient treatment as well as treatment in the hospital has been continuously promoted with the development of hospital information technology. And sharing of information using PHR drawn from individuals allows self-health care of various health institutions and individuals [2]. Initially, patients should first should objectively observe their health condition in doing self-management for health promotion and then clinicians should provide guidelines. Therefore, services that enable individuals to intuitively check their overall health condition including their behavior, diet and work out-related information is required beyond the primary information provided by medical service providers through visualization of PHR. Furthermore, additional installation of DSS (Decision Support System) that provides guidelines for decision making of 
personal health management within the service providing visualized PHR information is required. The PHR will have a positive effect in understanding and legibility when information is expressed by distinguishing characteristics of users and applying personalized visualization technique. However, the design of services that provides personalized PHR information to users has not yet been diversified. In addition, PHR can be summarized as a service that helps consumers to open their medical information anytime, anywhere, and directly enter and manage their health information to support lifelong health care. Currently, information system in the health care field is building and operating various systems to meet the increasing demands for the health and medical and the budget for informatization is increasing accordingly. However, the current systems are not linked even though they are interrelated between various systems, causing duplicated or manual processing. In addition, only internal information of each institution is mutually connected and only part is connected or all of it is disconnected in sharing information, showing that consumer-centered PHR service such as management of personal history is not sufficient. Furthermore, standardization or real-time network for information linking with each institution is not prepared yet, causing problems for insufficient real-time provision of various data such as data on current state.

Standards related to PHR can be divided into three areas which are the exchange of PHD (Personal Health Device) information, the exchange of medical information devices and the exchange of information on IoT (Internet of Things) device. First, the Institute of Electrical and Electronics Engineers (IEEE) 11073 was enacted in the field of personal health devices. It is distributed as an ISO international standard according to International Organization for Standardization (ISO) and standard harmonization between IEEEs. The ISO/IEEE 11073 PHD Group is continuously enacting standards for each personal health device based on standard protocols (ISO/IEEE 11073-20601) to measure and transfer personal biometrics. The exchange of PHR-based medical information is necessary to deliver personal health information such as medical records and checkup results saved in medical institutions, and it is classified as follows. 


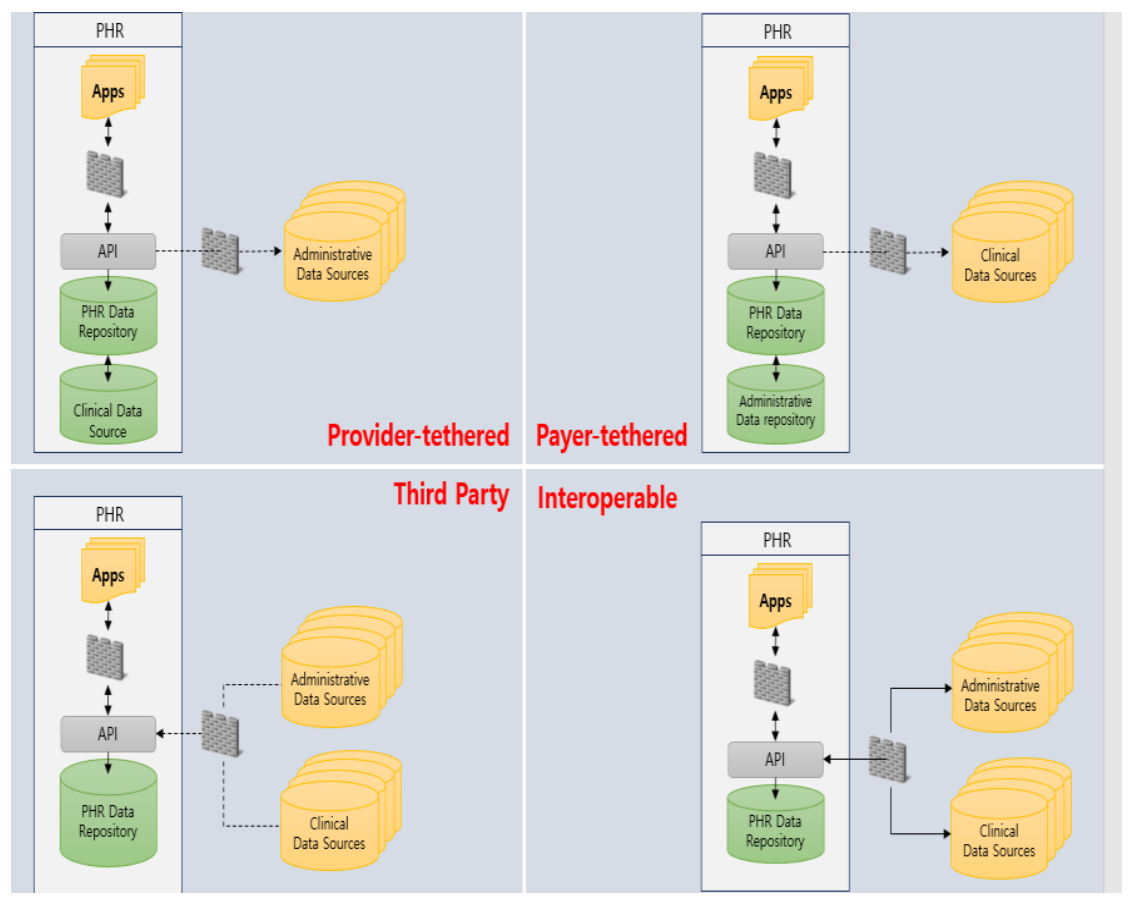

Fig. 1: Elements of Blockchain

This includes standards for terms, code system, protocol, and medical documents in the field of health and medical information. Standards such as SNOMED-CT and UMLS for terms and code systems, LOINC for checkup code, ICD for diagnosis code, RxNorm for medicine code are being used. In case of protocols, there are the $\mathrm{V} 2$ and V3, which are message standards for exchanging medical information, DICOM for transferring medical video image, CCD of HL7 as standard for medical documents and Consolidated CDA. Standardization is in progress related to healthcare including PHR in the field of IoT. The OIC has started to develop standards about healthcare applications and services in IoT environment by making Healthcare Task Group and showed showcase that works by linking with IoT standard platform and existing healthcare standards in one M2M in 2018. As wearable devices such as such as activity tracker, heart rate measurement, and various IoT devices such as sleep monitoring device and smart home devices are developed recently, it is expected that there would be active discussion and enactment for PHR related healthcare standards in the field of IoT in the future.

\section{Blockchain-based PHR System Framework using Cloud}

Medical industries can manage information such as medical records, accident history, constitution, diseases, and checkup history of individuals to enable more accurate medical care and provide better medical services accordingly. In addition, unnecessary duplicated checkup and the costs that come with it can be reduced 
through this kind of information management. This reduces the economic burden of patients and increases trust in medical treatment. As such, blockchain technology is expected to bring innovative changes in various fields including healthcare, distribution, insurance, and medical care.

Method to build blockchain-based PHR system is as follows. Basically, security is the most important in PHR. So, functions about this should be implemented. In this paper, it is operated in closed manner so that only approved users based on Cloud that is good to be used by users (medical institutions, patients, etc.) can use the system, and private key is provided to each user to approve access. This system allows users to create an API (Application Program Interface) to process and use data in desired form and gives flexibility to expand use environment when the data is getting big. Cloud is consisted of several layers, which are 'Layer 1' where basic information of patients is saved, 'Layer 2' where medical information within individual patient information is saved in the form of blockchain, and 'Layer 3' where information is saved as database that applies HL7 and KOSTOM standardized for each patient. There is a public key that can identify the patients in the Layer 1, acting as a role of address that can find the location of patients within the Cloud.

Layer 2 is consisted of several nodes and each node is designated by individual users such as medical institutions and pharmacy. When each user updates the block (updating information of patients), other nodes can also be connected to the chain by copying the same blocks. As such, it is consisted of multiple nodes and it makes it difficult to change information when an external attack occurs. This also increases the integrity of the information in the system. Layer 3 builds database through standardization by applying HL7 and KOSTOM, making it easy to be used by users when they want to check the medical information (PHR) of patients or use it in their research. 


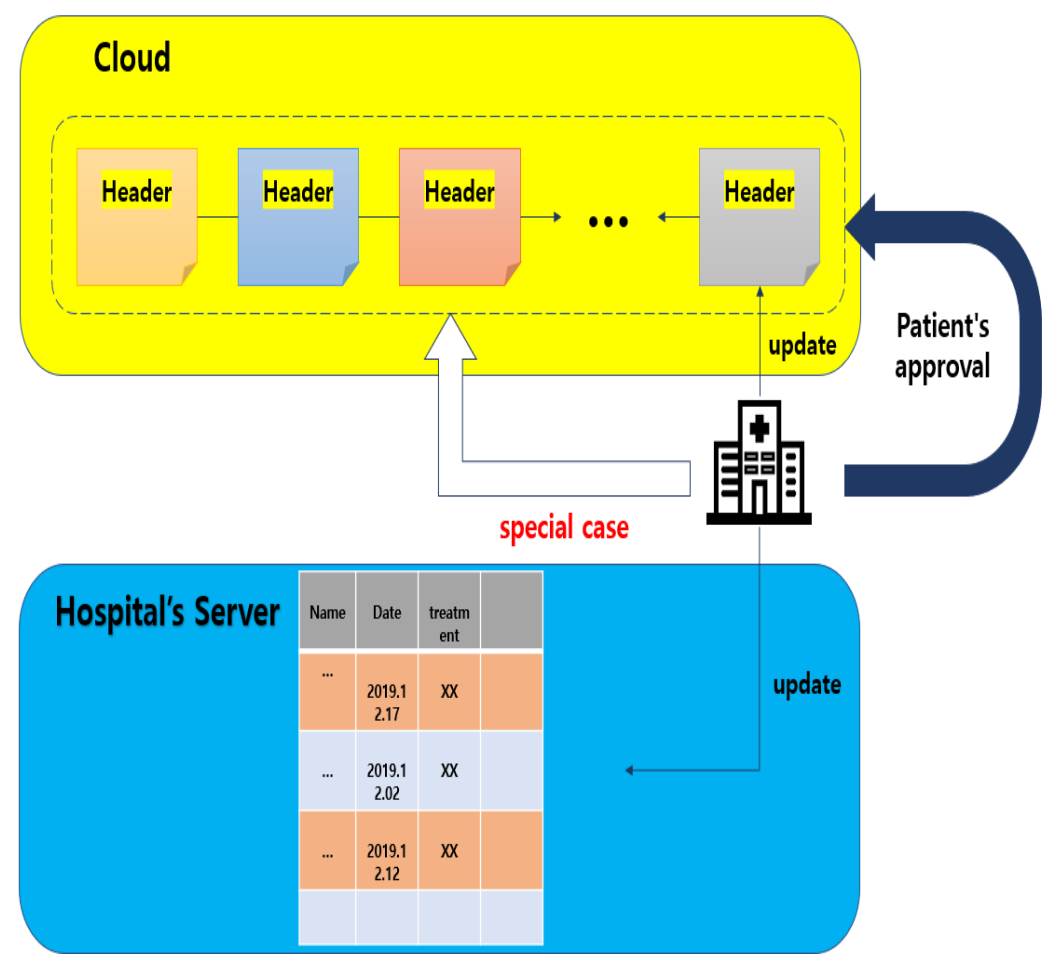

Fig. 2: Cloud-based PHR

When users who are authorized to update the medical information of patients update the block (patient information), they save the information on the cloud while the server saves the activity of the said users. In addition, users can update patient information in the cloud. The encrypted address is recorded in the information to be connected in the same blockchain of header (information of block), making it automatically connected and creating structure that prevent users from opening PHR of patients. There may be situation in which PHR of patients should be opened. At this time, PHR can be opened under the consent of patients. Sometimes, it is necessary to use medical information of patients in cloud DB for the purpose of research. For this case, users can use the API in the cloud to create new DB through the anonymizing process of $\mathrm{DB}$. The system will record the database access of the registered user in the database history as research purposes.

\section{Conclusion}

Blockchain technology can be used throughout the health and medical field. In particular, we need to pay attention to the fact which medical consumers, that is, individuals who were recognized as a victim of information asymmetry due to the medical information monopoly of hospitals can regain their sovereignty through blockchain technology. 
Current medical paradigm is shifting from treatment to management. In the management, it is important to know not only the current health condition of the patient but also the existing disease and its treatment process. In particular, the storage method of medical information is changing to PHR, an integrated record of EMR information with additional medical information of patients, beyond the environment where medical information is recorded and stored within EMR system. The emergence of PHR according to such change in the medical field makes it easy to understand the health condition of patients. Therefore, it is suitable for providing personalized medical services to patients.

\section{Acknowledgements}

Funding for this paper was provided by Namseoul University

\section{References}

Chiauzzi E., Rodarte C., and DasMahapatra P. (2015). Patient-centered activity monitoring in the self-management of chronic health conditions, BMC medicine, 13(1), 77 .

European Parliament, Union CotE. Regulation (EU) 2016/679 of the European Parliament and of the Council of 27 , on the protection of natural persons with regard to the processing of personal data and on the free movement of such data, and repealing Directive 95/46/EC (General Data Protection Regulation). 2016.

FCA (2017). Distributed Ledger Technology Feedback Statement on Discussion. Paper for the purposes of Regulation 2016/67.

Fragidis L. L. and Chatzoglou P. D. (2017), Development of nationwide electronic health record (nehr): An international survey. Health Policy and Technology, 6(2), 124-133.

Hölbl, M., Kompara, M., Kamišali'c, A., and Zlatolas, L.N. (2018). A Systematic Review of the Use of Blockchain in Healthcare. Symmetry.

Jang H. J., Kang M. W., and Chung Y. W. (2016). An Opportunistic Routing Protocol based on Contact Ratio. International Journal of Power Devices and Components for Smart Device, 3(1).

Kim H. G., Park J.T., and Moon IlY. (2018). Research for Applying Big Data System to Internet of Things devices using Web Technology. International Journal of Mobile Device Engineering, 2 (2), 2018. 
Lee S. Y. (2020) Blockchain for Medical Information of Personal Health Record System. Journal of Smart Technology Applications, 1(1).

Marlon, D., Marcello, L. R., Jan, M., and Hajo, A. R. (2018) Fundamentals of Business Process Management. Second Edition. Springer, 2018.

Naga Mallik Raj S., and Neeraja S. A Survey on Map Reduce Framework for Clustering Security. International Journal of Private Cloud Computing Environment and Management, 6(1).

Nakamoto S. (2008). Bitcoin: A peer-to-peer electronic cash system.

Park, Y., Lee, E., Na, W., Park, S., Lee, Y., and Lee, J. (2019) Is Blockchain Technology Suitable for Managing Personal Health Records? Mixed-Methods Study to Test Feasibility. Journal of Medical Internet Research, 21(2).

Randall D., Goel P., and Abujamra R. (2017) Blockchain applications and use cases in health information technology. Journal of Health Medical Information, 8(276), 2.

Richard, H., Vishal, S.B., Yi-Min, C., Alin, D., Fenno, F., Terry, H., and Victor, V. (2016). Towards a Shared Ledger Business Collaboration Language Based on DataAware Processes. Springer International Publishing Switzerland, Q.Z. Sheng et al. (Eds.), ICSOC 2016, LNCS 9936, 18-36.

Roehrs, A., da Costa, A., da Rosa Righi, R., et al. (2018) Analyzing the Performance of a Blockchain-based Personal Health Record Implementation. Journal of Biomedical Informatics, 92.

Sekhar Ch., and Suneetha N. (2018) A Study on Data Categorization for Data Analytics. International Journal of Internet of Things and Big Data. 3(1), 1-6.

Steve, H., Rituparna, B., Martin, W., Natalia, B. (2016). Internet of Things, Blockchain and Shared Economy Applications. Procedia Computer Science, 98, 2016.

Tuan T., A.D., Ji, W., Gang, C., Rui, L., Beng, C.O, and Kian-Lee, T. (2017) BLOCKBENCH: A Framework for Analyzing Bitfury group. Digital Assets on Public Blockchains, 2016 Private Blockchains, SIGMOD’17, Chicago, USA.

Yli-Huumo, J.; Ko, D.; Choi, S.; Park, S.; Smolander, K. (2016) Where Is Current Research on Blockchain Technology? A Systematic Review. PLoS ONE, 1-27. 
Zhang P., Walker M., White J., Schmidt D. C., and Lenz G. (2017) Metrics for assessing blockchain-based healthcare decentralized apps.

Zibin, Z., Shaoan, X., Hongning, D., Xiangping, C., and Huaimin, W. (2017) An Overview of Blockchain Technology: Architecture, Consensus, and Future Trends. IEEE 6th International Congress on Big Data.

Zimmer, D. (2017). Property Rights Regarding Data? Münster Colloquia on EU Law and the Digital Economy III, 101-8. 